\title{
A Simple Method to Improve the Accuracy of Advection in Discontinuous Galerkin Methods for Navier-Stokes Simulations
}

\author{
Eric Johnsen* Loc Khieu and Aditya Nair \\ University of Michigan, Ann Arbor, MI 48109, USA
}

\begin{abstract}
Discontinuous Galerkin (DG) methods have seen increased use over the past few decades. Recent work has shown that extremely accurate solutions $(3 p+2)$ can be achieved for pure diffusion problems when using Recovery Discontinuous Galerkin (RDG) methods. However, the order of accuracy for advection is at best $2 p+1$, thus negating the main advantages of RDG, particularly for advection-diffusion equations, such as the Navier-Stokes equations. In the present paper, we determine a new approach based on monotone upstream centered schemes for conservation laws (MUSCL) to improve the accuracy of advection. Furthermore, we show that RDG is more computationally efficient than certain commonly used diffusion methods, thus making RDG a more viable approach for high-fidelity turbulence simulations. For this purpose, we use three test problems. The interaction of two rarefactions is considered to evaluate convergence of the new advection approach. The problem of decaying isotropic turbulence with eddy shocklets is used to demonstrate the improvement of the proposed approach over standard advection. A pure diffusion Navier-Stokes problem is employed to compare the performance of RDG against other commonly used diffusion schemes.
\end{abstract}

\section{Introduction}

Discontinuous Galerkin (DG) methods have become popular methods to simulate convectiondominated problems, especially following developments by Cockburn and Shu. ${ }^{4-7}$ One of their main advantages lies in their super-convergence, at a rate of $2 p+1$, where $p$ is the order of the basis function in each cell. For diffusion, the recently developed Recovery-based DG ${ }^{16,17,21-23}$ approach provides an even more accurate scheme $(3 p+2)$; in addition, Recovery is unambiguous and stable, with no need for additional stabilization terms. For advection-diffusion problems, such as compressible turbulence simulations with the Navier-Stokes equations, advection would significantly limit the accuracy of the method: for instance, for $p=2$, advection would be fifthorder accurate, while diffusion would be eighth. Another limitation is that, while its accuracy is very high, Recovery has yet to be shown to be more efficient than other schemes.

Recovery has recently been used for direct numerical simulation of nearly incompressible and compressible turbulence, including shocks. ${ }^{12,13}$ While the temporal evolution of the kinetic energy and enstrophy were well-resolved on grids of $64^{3}$, that dilatation was not, particuarly in the more compressible simulations. It is suspected that lower accuracy of the advection scheme is responsible for this phenomenon.

In the present work, we seek to address the issues raised above, namely: (i) Devise a more accurate treatment of advection that remains is not excessively expensive computationally, and (ii)

*Assistant Professor, Mechanical Engineering Department, AIAA Member. 
assess the efficiency of Recovery compared to other common diffusion schemes. For the first point, we draw from our experience with Recovery. The underlying principle behind Recovery is akin to $P_{M} P_{N}[9,10$,$] and Hermite [18,19$,$] finite volume methods, in which high order is achieved by$ expanding the stencil and carrying several degrees of freedom within a cell. $P_{M} P_{N}$ methods are storage-friendly and may be more flexible for certain types of problems. However, it is not clear whether the optimal order of accuracy is achieved in these methods. Thus, we choose to pursue a different approach. The paper is organized as follows. Section 1 describes the numerical approach, including Fourier analysis, and Section Щ1I presents the results for two interacting rarefactions and compressible turbulence. In the appendix, we include comparisons between different DG schemes for diffusion using a pure diffusion Navier-Stokes problem.

\section{Numerical methodology}

The full three-dimensional compressible Navier-Stokes equations with heat conduction for a calorically perfect gas and a power-law viscosity form the governing equations. The description of the enhanced Recovery procedure for DG simulations of compressible turbulence is omitted to prevent redundancies. The diffusion scheme is based on Recovery, and advection is treated in a solution-adaptive fashion, such that hierarchical limiting ${ }^{15,24}$ is applied locally, only near discontinuities. This approach prevents numerical dissipation from overwhelming the small turbulent scales. The reader is referred to past work on this topic for additional information. ${ }^{12,13}$

\section{A. Combining DG and MUSCL}

Using Recovery for the diffusive terms, accuracy of the order $3 p+2$ in the cell-average of the conservative quantities are readily achieved by using information from the neighboring cells. However, the formal order of accuracy of the convective terms is only $2 p+1$. Even though Recovery leads to high order for the diffusive terms, the smoothness of the recovered function makes it unusable for advection, where numerical dissipation is required for stability. Hence, to improve the order by at least one, a monotone upstream centered scheme for conservation laws (MUSCL) approach ${ }^{20}$ that uses information from the neighboring cell is developed. This approach does not violate the locality of DG since the stencil is not increased and only information from adjacent cells is required.

Assume solution in cell $\Omega_{j}$ is represented by a $p^{\text {th }}$-order polynomial,

$$
u_{j}(x)=\sum_{\alpha=0}^{p} C_{\alpha x, j} \phi_{\alpha, j} x .
$$

In the DG+MUSCLapproach, the interface values required for flux calculation are calculated instead from the enhanced $\hat{u}_{j}(x)$, which is a polynomial of degree $p+1$,

$$
\hat{u}_{j}(x)=\sum_{\alpha=0}^{p+1} C_{\alpha x, j} \phi_{\alpha, j} x=u_{j}(x)+C_{(p+1) x, j} \phi_{p+1, j} x .
$$

The value of $C_{(p+1) x, j}$ is computed from the forward difference

$$
\Delta_{+} C_{p x, j}=C_{p x, j+1}-C_{p x, j}
$$

and the backward difference

$$
\Delta_{-} C_{p x, j}=C_{p x, j}-C_{p x, j-1}
$$

as follow

$$
\begin{gathered}
C_{(p+1) x, j}=\frac{1}{2(2 p+1)} \times \frac{\Delta_{+} C_{p x, j}+\Delta_{-} C_{p x, j}}{2} . \\
2 \text { of } 10
\end{gathered}
$$


Near discontinuities, the value calculated from [3] will be replaced by a limited one with an appropriate limiter, e.g, minmod:

$$
C_{(p+1) x, j}=\frac{1}{2(2 p+1)} \operatorname{minmod}\left(\Delta_{+} C_{p x, j}, \Delta_{-} C_{p x, j}, \frac{1}{2}\left(\Delta_{+} C_{p x, j}+\Delta_{-} C_{p x, j}\right)\right),
$$

with minmod function is defined by

$$
\operatorname{minmod}(a, b, c)=\min (|a|,|b|,|c|) \times \frac{\operatorname{Sgn}(a)+\operatorname{Sgn}(b)}{2} \times\left|\frac{\operatorname{Sgn}(b)+\operatorname{Sgn}(c)}{2}\right| .
$$

This approach has the advantage of adding an extra degree of freedom in a cell without having to solve additional transport equations for that coefficient. Therefore, accuracy is enhanced without significant increase in computational expense. However, the overall computational stencil grows to five cells, from the original three-cell stencil.

It should be noted that the reconstructed $\hat{u}_{j}$ will not be used in the discretization of the diffusion terms. Fourier analysis shows that the order of accuracy of DG+MUSCL increases by one comparing to that of the original DG.

\section{B. Fourier analysis for DG+MUSCL}

In this section, we perform the Fourier analysis for the DG+MUSCLmethod presented in $\mathrm{A}$ The linear spatial operator is obtained by discretization of the scalar linear advection equation in one dimension,

$$
\partial_{t} u=-\partial_{x} F(u) \Leftrightarrow \partial_{t} u=-a \partial_{x} u
$$

Without loss of generality, we may assume a positive constant advection speed, $a>0$. Thus, an upwind flux at the interface is

$$
F=F\left(u_{L}, u_{R}\right)=a u\left(u_{L}, u_{R}\right)=a u_{L}
$$

The DG update equations have the following general form:

$$
\begin{gathered}
\partial_{t} \int_{\Omega_{i}} v_{j} u_{j} d x=-\int_{\Omega_{j}} v_{j} a \partial_{x} u_{j} d x \\
\Rightarrow \partial_{t} \int_{\Omega_{j}} v_{j} u_{j} d x=-a\left[v_{j}\left(x_{j+1 / 2}\right) u_{j+1 / 2}-v_{j}\left(x_{j-1 / 2}\right) u_{j-1 / 2}\right]+a \int_{\Omega_{j}} u_{j} \partial_{x} v_{j} d x
\end{gathered}
$$

where interface values are calculated by

$$
\begin{gathered}
u_{j-1 / 2}=u\left(\hat{u}_{j-1, R}, \hat{u}_{j, L}\right)=\hat{u}_{j-1, R}=\hat{u}_{j-1}\left(x_{j-1 / 2}\right), \\
u_{j+1 / 2}=u\left(\hat{u}_{j, R}, \hat{u}_{j+1, L}\right)=\hat{u}_{j, R}=\hat{u}_{j}\left(x_{j+1 / 2}\right)
\end{gathered}
$$

and $v_{j}$ is test functions.

We make the DG scheme nondimensional by multiplying both sides of $\mathbf{7}$ with $\Delta x / a$, then we determine the Fourier transform of the spatial operator. It is a $(p+1) \times(p+1)$ matrix $\hat{M}(\beta)$, with $\beta$ is the frequency variable. Next we calculate its eigenvalues; there are $p+1$ of these but we are only interested in the one consistent with the exact eigenvalue $\lambda_{\text {exact }}$ for $\beta \rightarrow 0$; we'll call this value $\lambda_{\text {con }}$. The Fourier transform of the nondimensionalized spatial operator in the original PDE (6) is

$$
\lambda_{\text {exact }}=-i \beta
$$


The leading term of the Taylor expansion of $\lambda_{\text {con }}$ should be identical, and the ratio between $\beta$ powers of the leading error term and the exact eigenvalue indicates the order of accuracy - in other words, the order of accuracy is one less than the power of $\beta$ in the leading error term.

As an example, consider DG+MUSCLfor $p=1$. Solution in an arbitrary cell $\Omega_{j}$ is represented by a polynomial of degree one,

$$
u_{j}(x)=\sum_{\alpha=0}^{1} C_{\alpha x, j} \phi_{\alpha, j} x=C_{0 x, j} \phi_{0, j}+C_{1 x, j} \phi_{1, j}=C_{0 x, j}+C_{1 x, j}(2 \xi-1),
$$

and its enhanced representation is

$$
\hat{u}_{j}(x)=\sum_{\alpha=0}^{2} C_{\alpha x, j} \phi_{\alpha, j} x=C_{0 x, j}+C_{1 x, j}(2 \xi-1)+C_{2 x, j}\left(6 \xi^{2}-6 \xi+1\right) .
$$

The local spatial variable $\xi \in[0,1]$ relates to the global coordinate by

$$
\xi=\frac{x-x_{j}}{\Delta x_{j}}-\frac{1}{2}
$$

subscript $j$ in $\Delta x_{j}$ will be subsequently omitted because the analysis is performed on a uniform grid. Coefficient $C_{2 x, j}$ is calculated by 3 ,

$$
C_{2 x, j}=\frac{1}{12}\left(\Delta_{+} C_{1 x, j}+\Delta_{-} C_{1 x, j}\right)=\frac{1}{12}\left(C_{1 x, j+1}-C_{1 x, j-1}\right) ;
$$

the unique values at the left and right interfaces are computed using 8

$$
\begin{gathered}
u_{j-1 / 2}=\hat{u}_{j-1}\left(x_{j-1 / 2}\right)=C_{0 x, j-1}+C_{1 x, j-1}+\frac{1}{12}\left(C_{1 x, j}-C_{1 x, j-2}\right), \\
u_{j+1 / 2}=\hat{u}_{j}\left(x_{j+1 / 2}\right)=C_{0 x, j}+C_{1 x, j}+\frac{1}{12}\left(C_{1 x, j+1}-C_{1 x, j-1}\right) .
\end{gathered}
$$

The two update equations for $p=1$ are obtained by substituting appropriate values to 7 .

$$
\frac{\Delta x}{a} \frac{\partial}{\partial t}\left[\begin{array}{l}
C_{0 x, j} \\
C_{1 x, j}
\end{array}\right]=\underbrace{\left[\begin{array}{cc}
-1+T^{-1} & -\frac{11}{12}+\frac{13}{12} T^{-1}-\frac{1}{12} T^{-2}-\frac{1}{12} T \\
3\left(1-T^{-1}\right) & -\frac{13}{4}-\frac{11}{4} T^{-1}+\frac{1}{4} T^{-2}-\frac{1}{4} T
\end{array}\right]}_{M(T)}\left[\begin{array}{c}
C_{0 x, j} \\
C_{1 x, j}
\end{array}\right]
$$

here $T$ represents forward translation by one cell, thus

$$
T C_{\alpha x, j}=C_{\alpha x, j+1}
$$

and

$$
T^{-1} C_{\alpha x, j}=C_{\alpha x, j-1} .
$$

The Fourier transform of operator $M(T)$ is

$$
\hat{M}(\beta)=\left[\begin{array}{cc}
-1+e^{-i \beta} & -\frac{11}{12}+\frac{13}{12} e^{-i \beta}-\frac{1}{12} e^{-2 i \beta}-\frac{1}{12} e^{i \beta} \\
3\left(1-e^{-i \beta}\right) & -\frac{13}{4}-\frac{11}{4} e^{-i \beta}+\frac{1}{4} e^{-2 i \beta}-\frac{1}{4} e^{-i \beta}
\end{array}\right] .
$$

It has two eigenvalues, of which closed forms are too complicated to display; their Taylor expansion are:

$$
\begin{aligned}
& \lambda_{1}=-6+2 i \beta+O\left(\beta^{2}\right), \\
& \lambda_{2}=-i \beta-\frac{i \beta^{5}}{720}+O\left(\beta^{6}\right) .
\end{aligned}
$$

It is obvious that $\lambda_{2}$ is the consistent eigenvalue, which represents the exact eigenvalue (9) with a fourth-order accuracy, one order higher than that of the standard DG discretization for advection. 


\section{Results}

Three two problems are considered. The interaction of two rarefactions is first considered to evaluate convergence of the new advection approach. Then, the problem of decaying isotropic turbulence with eddy shocklets is used to demonstrate the improvement of the proposed approach over standard advection. In the appendix, a pure diffusion Navier-Stokes problem is employed to compare the performance of Recovery against other commonly used diffusion schemes. Recovery is more efficient than several other popular diffusion approaches.

\section{A. Rarefactions interaction}

We consider the one-dimensional problem of left- and right-running rarefactions smoothly interacting with each other for the Euler equations (no diffusion), to test the improved advection treatment compared to the traditional advection scheme. The problem is isentropic and errors in entropy are used to establish the order of accuracy. Tables 1 and 2] show the convergence of the traditional and MUSCL-based schemes. The traditional advection schemes exhibit more or less $2 p+1$ convergence rates, as expected; for $p=1$, a slightly better rate is obtained, while for $p=2$ it is slightly worse. The MUSCL-based approach yields similar superconvergent results, though the $p=1$ case produces significantly better results. While promising, the results are better than the theory (Fourier analysis) predicts. We are currently investigating the reasons for this behavior.

\section{B. Compressible turbulence}

The decaying compressible isotropic turbulence problem ${ }^{14}$ is adapted here to evaluate the performance of the present Recovery method. In this problem, the turbulent Mach number $M_{t}$ is sufficiently high that weak shocks (eddy shocklets), produced by the turbulent motions, form spontaneously. In order to produce strong and frequent shocklets over the whole domain, a high turbulent Mach number is considered $\left(M_{t}=0.7\right)$. This problem tests the capability of applying limiting in randomly distributed regions based on the discontinuity sensor. The detailed initial conditions are described in Johnsen et al.;1 the only difference lies in the turbulent Mach number.

In past work, ${ }^{12}$ it could be observed that, while kinetic energy and enstrophy were well resolved already on grids of $64^{3}$ with the standard approach for advection, dilatation was not. It is interesting to note that such a feature has been observed with other approaches for compressible turbulence. ${ }^{11}$

Table 1. Convergence of the advection treatment for the rarefactions interaction problem for $p=1$ vs. $p=0+$ MUSCL.

\begin{tabular}{lccc}
\hline Method & $N$ & $L_{2}$ error & $L_{2}$ order \\
\hline$p=1$ & 20 & $4.17 \mathrm{e}-4$ & - \\
& 40 & $3.90 \mathrm{e}-5$ & 3.42 \\
& 80 & $3.93 \mathrm{e}-6$ & 3.31 \\
$p=0+$ MUSCL & 160 & $3.60 \mathrm{e}-7$ & 3.45 \\
& 320 & $3.18 \mathrm{e}-8$ & 3.50 \\
& 20 & $2.59 \mathrm{e}-3$ & - \\
& 40 & $3.49 \mathrm{e}-4$ & - \\
& 80 & $3.85 \mathrm{e}-5$ & 2.89 \\
& 160 & $4.04 \mathrm{e}-6$ & 3.18 \\
& 320 & $4.22 \mathrm{e}-7$ & 3.26 \\
\hline
\end{tabular}




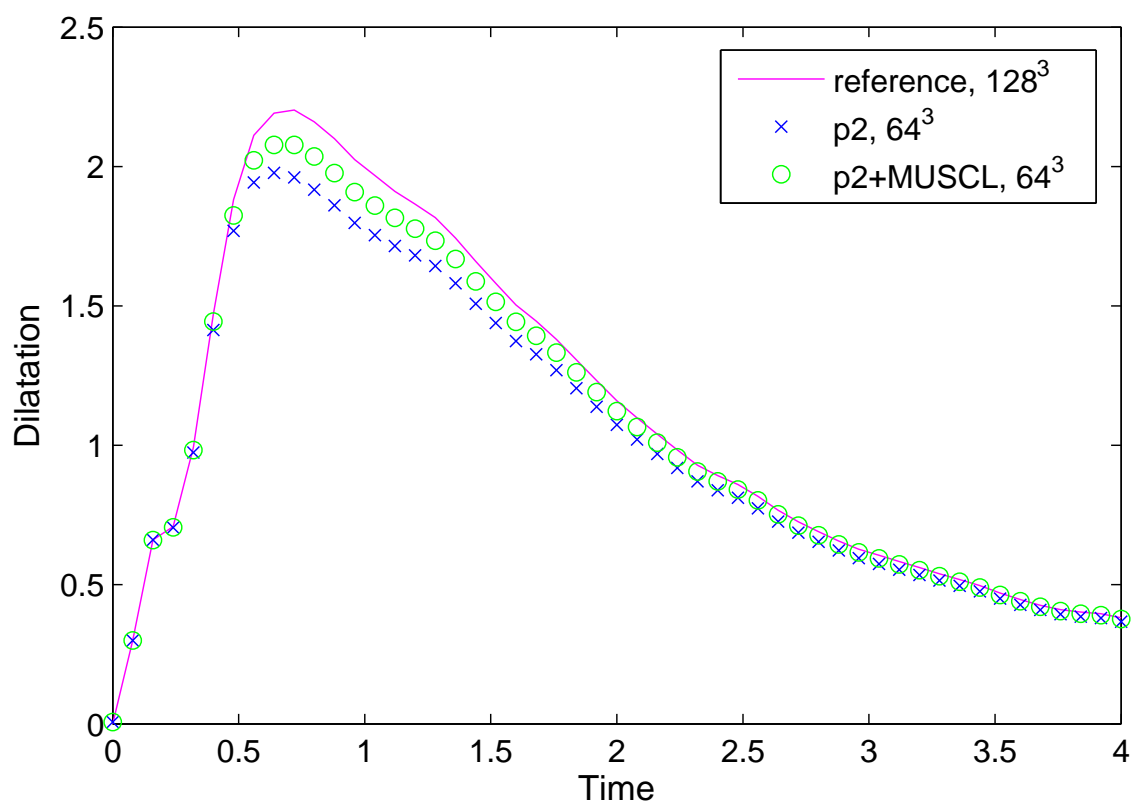

Figure 1. Temporal evolution of dilatation in the compressible isotropic turbulence problem with $M_{t}=0.7$ on $64^{3}$.

Figs. 1] and 2 compares the temporal evolution of dilatation for the two different approaches on different grids. With the present approach, dilatation is better represented on both grids. Although not shown here, the solution on $128^{3}$ is similar for both cases.

\section{Conclusions}

In the present paper, we propose a new approach based on MUSCL to improve the accuracy for advection and compare the efficiency of the Recovery-based Discontinuous Galerkin method for diffusion. We show that the accuracy of the convective terms can be improved by using a MUSCL

Table 2. Convergence of the advection treatment for the rarefactions interaction problem for $p=2$ vs. $p=1+$ MUSCL.

\begin{tabular}{lccc}
\hline Method & $N$ & $L_{2}$ error & $L_{2}$ order \\
\hline$p=2$ & 20 & $9.31 \mathrm{e}-5$ & - \\
& 40 & $8.83 \mathrm{e}-6$ & 3.40 \\
& 80 & $4.85 \mathrm{e}-7$ & 4.19 \\
$p=1+$ MUSCL & 160 & $2.10 \mathrm{e}-8$ & 4.53 \\
& 320 & $9.50 \mathrm{e}-10$ & 4.47 \\
& 20 & $7.37 \mathrm{e}-5$ & - \\
& 40 & $2.71 \mathrm{e}-6$ & 4.77 \\
& 80 & $1.15 \mathrm{e}-7$ & 4.55 \\
& 160 & $3.20 \mathrm{e}-9$ & 5.17 \\
& 320 & $6.54 \mathrm{e}-11$ & 5.61 \\
\hline
\end{tabular}

6 of 10 


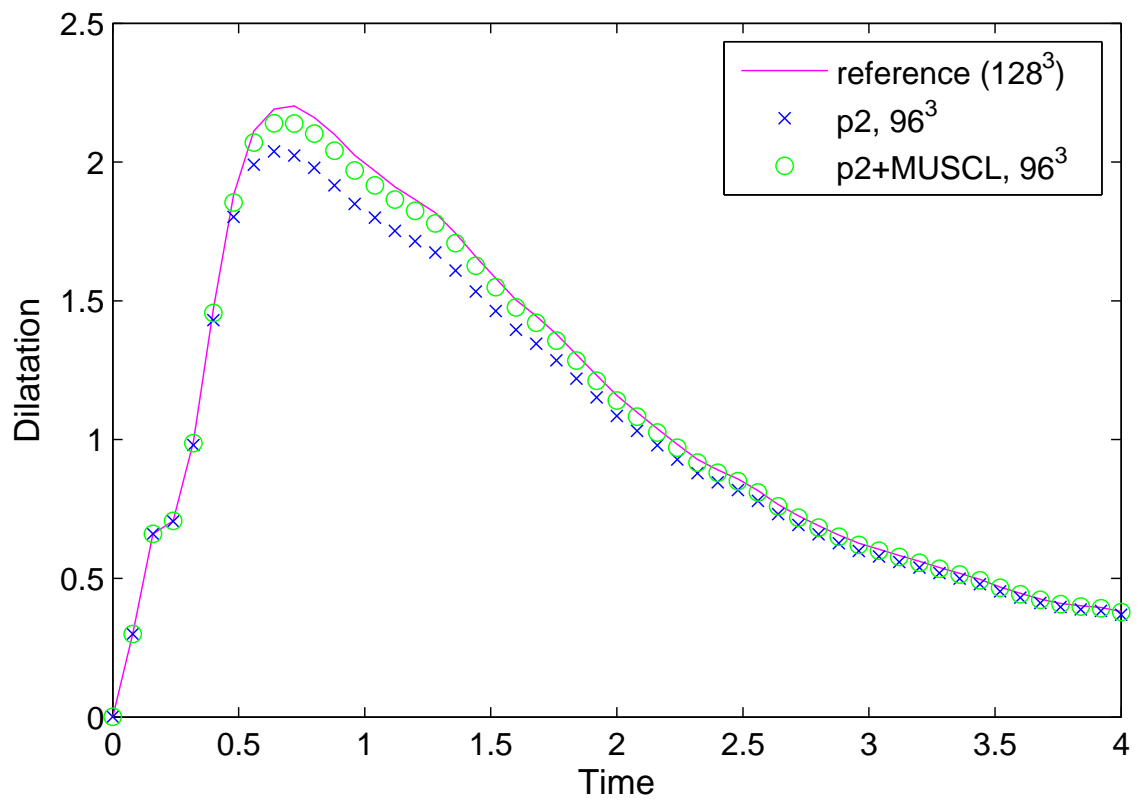

Figure 2. Temporal evolution of dilatation in the compressible isotropic turbulence problem with $M_{t}=0.7$ on $96^{3}$.

approach to expand the solution space within a cell. Several test problems are used to verify our approach. The interaction of two rarefactions is considered to evaluate convergence of the new advection approach. The problem of decaying isotropic turbulence with eddy shocklets is used to demonstrate the improvement of the proposed approach over standard advection. While promising, the results are better than the theory (Fourier analysis) predicts; we are currently investigating the reasons for this behavior.

Improving the order of accuracy of the advection terms reduces the discrepancy with diffusion, and thus makes the overal Recovery approach more viable for turbulence simulations. We expect that, thanks to its compact stencil and accuracy even in complex geometries, Recovery will offer significant advantages for high-fidelity (direct numerical simulation and large-eddy simulation) simulation of turbulent flows. In view of this, we are currently evaluating different approaches to improving advection.

\section{Acknowledgments}

The authors thank Sreenivas Varadan and Bram van Leer for helpful conversations.

\section{Appendix}

A pure diffusion Navier-Stokes problem is employed to compare the performance of Recovery against other commonly used diffusion schemes. While the extremely high order of accuracy of Recovery constitutes one of its main advantages, a possible limitation lies in its computational cost. In this section, we compare the efficiency of Recovery with other methods, primarily with the commonly used BR2 ${ }^{2,3}$ scheme using a two-dimensional pure diffusion Navier-Stokes problem. ${ }^{12}$ Fig. 3 shows convergence analysis for Recovery, BR2 and the interior penalty (IP) method $^{1}$ for $p=1,2$ and 3 at a fixed Von Neumann Number (VNN). Tables 3 , 4 and 5 show the relative cost 


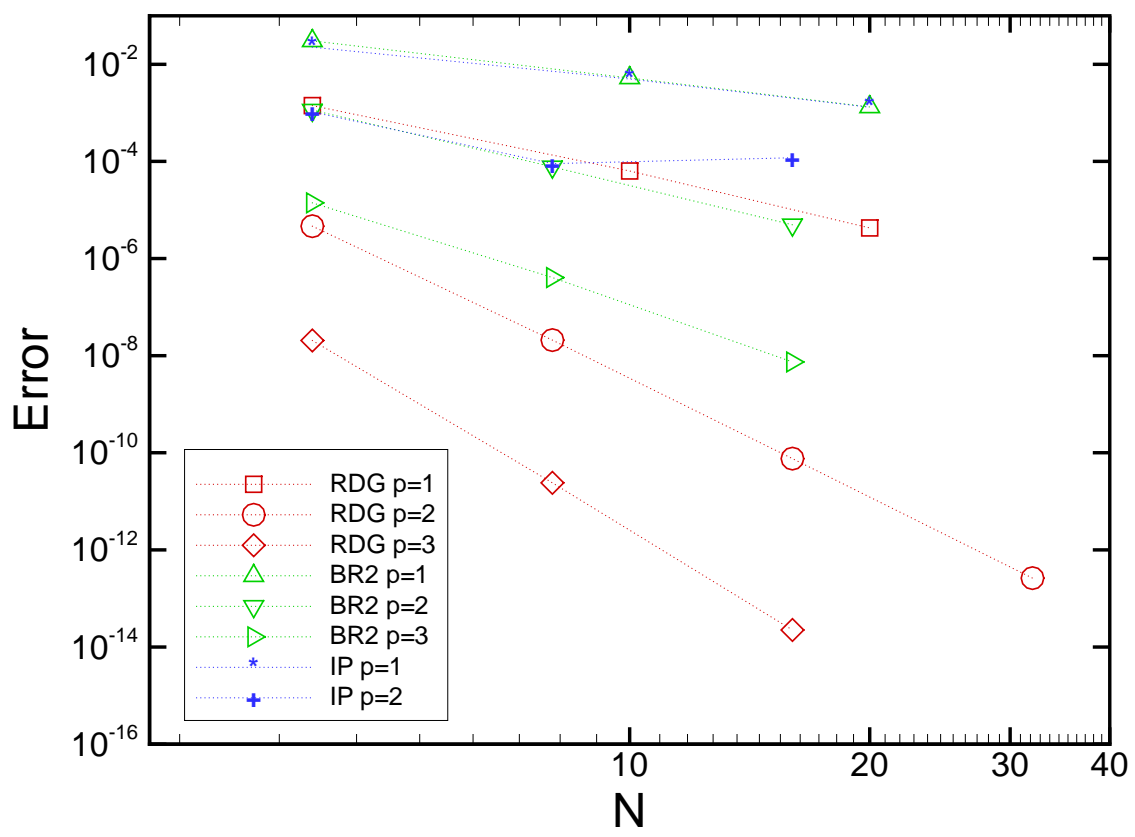

Figure 3. Error vs. number of grid points for the different diffusion schemes for the pure diffusion Navier-Stokes problem.

between Recovery, BR2 and IP, for a fixed VNN on an $N^{2}=8^{2}$ grid. Table [ shows the maximum VNN that each method can use. Given that the cost per time step is not very different between the methods and that the critical VNN is much larger for Recovery, it is clear that Recovery is the most efficient approach for diffusion considered here. It should be noted that IP runs had difficulty completing for grids larger than $8^{2}$ or $p=3$.

Table 3. Relative cost between Recovery, BR2 and IP on an $N^{2}=8^{2}$ grid for the pure diffusion Navier-Stokes problem for $p=1$.

\begin{tabular}{lc}
\hline Method & Time (normalized by Recovery) \\
\hline Recovery & 1.00 \\
BR2 & 1.33 \\
IP & 1.17 \\
\hline
\end{tabular}

Table 4. Relative cost between Recovery, BR2 and IP on an $N^{2}=8^{2}$ grid for the pure diffusion Navier-Stokes problem for $p=2$.

\begin{tabular}{lc}
\hline Method & Time (normalized by Recovery) \\
\hline Recovery & 1.00 \\
BR2 & 1.20 \\
IP & 1.05 \\
\hline
\end{tabular}




\section{References}

${ }^{1}$ D. N. Arnold, F. Brezzi, B. Cockburn and L. D. Marini, Unified analysis of discontinuous Galerkin methods for elliptic problems. SIAM J. Numer. Anal. 39 (2002) 1749-1779.

${ }^{2}$ F. Bassi, S. Rebay, A high-order accurate discontinuous finite element method for the numerical solution of the compressible Navier-Stokes equations, J. Comput. Phys., 131 (1997) 267-279.

${ }^{3}$ F. Bassi, S. Rebay, G. Mariotti, S. Pedinotti, M. Savini, A high-order accurate discontinuous finite element method for viscous and turbomachinery flows, in: Proceedings of the 2nd European Conference on turbomachinery Fluid Dynamics and Thermodynamics, Belgium 1997, 99-108.

${ }^{4}$ B. Cockburn and C.W. Shu, TVB Runge-Kutta local projection discontinuous Galerkin finite element method for scalar conservation laws II: General framework, Math. Comp. 52 (1989) 411-435.

${ }^{5}$ B. Cockburn, S. Y. Lin, and C. W. Shu, TVB Runge-Kutta local projection discontinuous Galerkin finite element method for conservation laws III: One dimensional systems, J. Comput. Phys. 84, 90 (1989).

${ }^{6}$ B. Cockburn, S. Hou and C. W. Shu, TVB Runge-Kutta local projection discontinuous Galerkin finite element method for conservation laws IV: One dimensional systems: The multidimensional case, Math. Comp. 54 (1990) $545-581$.

${ }^{7}$ B. Cockburn and C. W. Shu, The Runge-Kutta discontinuous Galerkin method for conservation laws V: Multidimensional systems, J. Comput. Phys. 141 (1998) 199-224.

${ }^{8}$ B. Cockburn, C. W. Shu, Runge-Kutta discontinuous Galerkin methods for time-dependent convection-diffusion systems, SIAM J. Numer. Anal., 35 (1998) 2440-2463.

${ }^{9}$ M. Dumbser, D. S. Balsara, E. F. Toro and C. D. Munz, A unified framework for the construction of one-step finite volume and discontinuous Galerkin Schemes on unstructured meshes, J. Comput. Phys. 227 (2008) 8209-8253.

${ }^{10}$ M. Dumbser, Arbitrary high order $P_{N} P_{M}$ schemes on unstructured meshes for the compressible Navier-Stokes equations, Comput. Fluids 39 (2010) 60-76.

${ }^{11}$ E. Johnsen, J. Larsson, A. V. Bhagatwala, W. H. Cabot, P. Moin, B. J. Olson, P. S. Rawat, S. K. Shankar, B.

Table 5. Relative cost between Recovery, BR2 and IP on an $N^{2}=8^{2}$ grid for the pure diffusion Navier-Stokes problem for $p=3$.

\begin{tabular}{lc}
\hline Method & Time (normalized by Recovery) \\
\hline Recovery & 1.00 \\
BR2 & 1.12 \\
IP & N/A \\
\hline
\end{tabular}

Table 6. Maximum VNN for each method, for the pure diffusion Navier-Stokes problem.

\begin{tabular}{lccc}
\hline Method & $p$ & $N$ & $\mathrm{VNN}$ \\
\hline Recovery & 1 & $4^{2}$ & 1.00 \\
& 2 & $4^{2}$ & 0.48 \\
& 3 & $4^{2}$ & 0.25 \\
& 1 & $8^{2}$ & 0.90 \\
& 2 & $8^{2}$ & 0.40 \\
& 3 & $8^{2}$ & 0.25 \\
\hline BR2 & 1 & $4^{2}$ & 0.165 \\
& 2 & $4^{2}$ & 0.039 \\
& 3 & $4^{2}$ & 0.016 \\
& 1 & $8^{2}$ & 0.150 \\
& 2 & $8^{2}$ & 0.039 \\
& 3 & $8^{2}$ & 0.016 \\
\hline
\end{tabular}

9 of 10 
Sjogreen, H. C. Yee, X. Zhong and S. K. Lele, Assessment of high resolution methods for numerical simulations of compressible turbulence, J. Comput. Phys. 228 (2010) 1213-1237.

${ }^{12}$ E. Johnsen, S. Varadan and B. van Leer, A three-dimensional recovery-based discontinuous Galerkin method for turbulence simulations, AIAA paper (2013) 2013-0515.

${ }^{13}$ E. Johnsen, S. Varadan and A. Nair, Recovery Discontinuous Galerkin Method for Compressible Turbulence, AIAA paper (2013) AIAA paper (2013) 2013-3066.

${ }^{14}$ S. Lee, S. K. Lele and P. Moin, Eddy shocklets in decaying compressible turbulence, Phys. Fluids A 3 (1991) 657-664.

${ }^{15}$ Y. Liu, C. W. Shu, E. Tadmor and M. Zhang, Central discontinuous Galerkin methods on overlapping cells with a nonoscillatory hierarchical reconstruction, SIAM J. Numer. Anal. 45 (2007) 2442-2467.

${ }^{16}$ M. Lo and B. van Leer, Analysis and Implementation of Recovery-Based Discontinuous Galerkin for Diffusion, AIAA paper (2009) 2009-3786.

${ }^{17}$ M. Lo and B. van Leer, Recovery-based discontinuous Galerkin for Navier-Stokes viscous terms, AIAA paper (2011) 2011-3406.

${ }^{18}$ J. Qiu and C. W. Shu, Hermite WENO schemes and their application as limiters for Runge-Kutta discontinuous Galerkin method: one-dimensional case, J. Comput. Phys. 193 (2003) 115-135.

${ }^{19}$ J. Qiu and C. W. Shu, Hermite WENO schemes and their application as limiters for Runge-Kutta discontinuous Galerkin method II: two-dimensional case, Comput. Fluids 34 (2005) 642-663.

${ }^{20}$ B. van Leer, Towards the ultimate conservative difference scheme, V. A second order sequel to Godunov's method, J. Comput. Phys. 32 (1979) 101-136.

${ }^{21}$ B. van Leer and S. Nomura, Discontinuous Galerkin for diffusion, AIAA paper (2005) 2005-5108.

${ }^{22}$ B. van Leer, M. Lo and M. van Raalte, A discontinuous Galerkin method for diffusion based on recovery, AIAA paper (2007) 2007-4083.

${ }^{23}$ B. van Leer and M. Lo, Unification of discontinuous Galerkin methods for advection and diffusion, AIAA paper (2009) 2009-400.

${ }^{24}$ Z. Xu, Y. Liu and C. W. Shu, Hierarchical reconstruction for discontinuous Galerkin methods on unstructured grids with a WENO-type linear reconstruction and partial neighboring cells, J. Comput. Phys. 228 (2009) $2194-2212$. 
This article has been cited by:

1. Loc H. Khieu, Krzysztof Fidkowski, Eric JohnsenAnalysis of Discontinuous Galerkin Approaches for Advection--Diffusion Problems . [Citation] [PDF] [PDF Plus] 